\title{
Synthesis and characterization of NiPcTSTNa(L) thin films
}

\author{
M E SÁNCHEZ-VERGARA ${ }^{\mathrm{a}, *}$, V GARCÍA-MONTALVO ${ }^{\mathrm{b}}$, J SANTOYO-SALAZAR ${ }^{\mathrm{c}}$, \\ R J FRAGOSO-SORIANO ${ }^{c}$ and O JIMÉNEZ-SANDOVAL ${ }^{\mathrm{d}}$ \\ ${ }^{a}$ Facultad de Ingeniería, Universidad Anáhuac México Norte, Avenida Universidad Anáhuac 46, Col. Lomas Anáhuac, \\ Huixquilucan, Estado de México 52786, México \\ ${ }^{b}$ Instituto de Química, Universidad Nacional Autónoma de México, Circuito Exterior S/N, Ciudad Universitaria, \\ México, D. F. 04510, México \\ ${ }^{\mathrm{c} C e n t r o ~ d e ~ I n v e s t i g a c i o ́ n ~ y ~ d e ~ E s t u d i o s ~ A v a n z a d o s ~ d e l ~ I n s t i t u t o ~ P o l i t e ́ c n i c o ~ N a c i o n a l, ~ U n i d a d ~ Z a c a t e n c o, ~}$ \\ Instituto Politécnico Nacional 2508, México, D. F. 07360, México \\ ${ }^{\mathrm{d} C e n t r o ~ d e ~ I n v e s t i g a c i o ́ n ~ y ~ d e ~ E s t u d i o s ~ A v a n z a d o s ~ d e l ~ I n s t i t u t o ~ P o l i t e ́ c n i c o ~ N a c i o n a l, ~ U n i d a d ~ Q u e r e ́ t a r o, ~}$ \\ Apartado Postal 1-798, Querétaro, Qro. 76001, México
}

MS received 8 September 2011

\begin{abstract}
NiPcTSTNa(L) [L = ethylenediamine (EDA); 1,4-diaminobutane (BDA); and 2,6-diamineanthraquinone (AqDA)] thin films were deposited by thermal evaporation. Their surface morphology was studied by AFM and SEM, and their chemical composition determined by EDS. Optical absorption studies of NiPcTSTNa(L) films were performed in the $200-1150 \mathrm{~nm}$ wavelength range. The optical bandgap of thin films was determined from the $(\alpha h v)^{1 / 2}$ vs $h v$ plots for indirect allowed transitions. The temperature dependence of electrical conductivity shows a semiconducting behaviour. The amorphous semiconductor films show thermal activation energies of electrical conduction between 3.3 and $3.7 \mathrm{eV}$.
\end{abstract}

Keywords. Thin films; semiconductors; optical absorption; electrical conductivity.

\section{Introduction}

Organic semiconducting materials are rapidly making an impact in the area of electronics and optoelectronics (Shafai and Anthopoulos 2001). Metallophthalocyanines (MPcs) are a prominent class of materials with electrical, optical and optoelectronic applications in many fields (de la Torre et al 2004; Campidelli et al 2008; Wojdyla et al 2008). One of the major advantages of using MPcs is their chemical stability as well as the ability to readily modify the molecular structure allowing the molecular engineering of their physical properties accordingly (Shafai and Anthopoulos 2001). High thermal stability of MPcs permits the deposition of high purity thin films through high-vacuum vapourization techniques (El-Nahass et al 2005a). Typically, the films exist as either $\alpha$ - or $\beta$-phases, or result in amorphous state depending mainly on the molecular self-stacking ability of the derivative, and also on the thin film fabrication procedure. The control of structure is of great importance in thin film technology since the main opto-electronic properties, such as the photogeneration of charge carriers, highly depend on the degree of molecular organization (Del Caño et al 2005). Because of the very good optical absorption of these molecules in the UV-vis region, there is considerable interest in the characterization of the electronic structures of phthalocyanines (El-Nahass et al 2005b). For thin

\footnotetext{
*Author for correspondence (elena.sanchez@anahuac.mx)
}

films of $\mathrm{H}_{2} \mathrm{Pc}, \mathrm{MgPc}, \mathrm{FePc}, \mathrm{CoPc}, \mathrm{CuPc}$ and $\mathrm{ZnPc}$, the visible and near ultraviolet absorption spectra have been measured (Davidson 1992). Schmeisser et al (1991) found that the lowest absorption band at $1.73 \mathrm{eV}$ is due to transitions between HOMO $\pi$ and LUMO $\pi *$ molecular orbitals which, due to the size of the $18-\pi$ electron systems, are well separated in energy from the molecular orbital structure of the phenyl and aza groups. They recorded a considerably smaller energy gap for $\mathrm{PbPc}$ than for $\mathrm{H}_{2} \mathrm{Pc}$ from the onset of the absorption spectrum, $1.2 \mathrm{eV}$. Bialek et al (2002) calculated the HOMO-LUMO gap of NiPc as $2.41 \mathrm{eV}$ and suggested that the orbitals next to the HOMO are separated from each other by $1.84,2.44,0.17,0.28$ and $0.09 \mathrm{eV}$, respectively and the energy level of the second unoccupied molecular orbital is widely separated from the LUMO, with differences becoming smaller for the following orbitals. Although NiPc has been the subject of various investigations, considerably less attention has been paid on its structural and optical characterization (El-Nahass et al 2005a). In this communication, we report the determination of optical parameters related to the main transitions in the UV-vis region, as well as the corresponding optical bandgap calculations for non-crystalline thin films based on nickel(II) phthalocyanine-tetrasulfonic acid tetrasodium salt (NiPcTSTNa) and axial diamine ligands: ethylenediamine (EDA), 1,4-diaminobutane (BDA) and 2,6-diamineanthraquinone (AqDA). The films were prepared by thermal evaporation and characterized by FT-IR spectroscopy, AFM, SEM, EDS and ellipsometry 
measurements. The refractive indices and absorption coefficients, parameters of particular interest for the design and fabrication of optoelectronic devices, have been determined for the films as well. Finally, temperature dependence of electrical conductivity has been investigated.

\section{Experimental}

The raw materials for this work were obtained from commercial sources and used without further purification. The characterization of the powder materials was carried out by FT-IR spectroscopy, in a Bruker spectrophotometer, model Tensor 27, as $\mathrm{KBr}$ pills in the 4000 to $300 \mathrm{~cm}^{-1}$ region. For the preparation of the films, a vacuum chamber was used with a diffusion pump and a special molybdenum crucible with a double-grid cover. A quartz fibre was put inside the crucible to avoid the ejection of grains towards the substrate at a temperature of $563 \mathrm{~K}$. The material was deposited on Corning 7059 glass substrates, which were ultrasonically degreased in warm methanol and dried in nitrogen, and on single-crystalline $c$-Si wafers, which were chemically etched with a $p$ solution $\left(10 \mathrm{ml} \mathrm{HF}, 15 \mathrm{ml} \mathrm{HNO}_{3}\right.$ and $300 \mathrm{ml} \mathrm{H}_{2} \mathrm{O}$ ) in order to remove the native oxide from the substrate surface. The base pressure used in the evaporation process was $10^{-5}$ torr at room temperature. The crucible-substrate distance was $20 \mathrm{~cm}$. For the SEM characterization of the films, a Jeol JSM 5200 CX microscope was used, at a $5 \mathrm{kV}$ potential for all samples. Topography and roughness of surfaces were analysed by atomic force microscopy (AFM), model Autoprobe CP Thermomicroscopes in Tapping mode. The thickness measurements were made with a Sloan Dektak IIA profilometer. The FT-IR characterization was carried out using a Nicolet 205 spectrophotometer, on Si substrate deposited

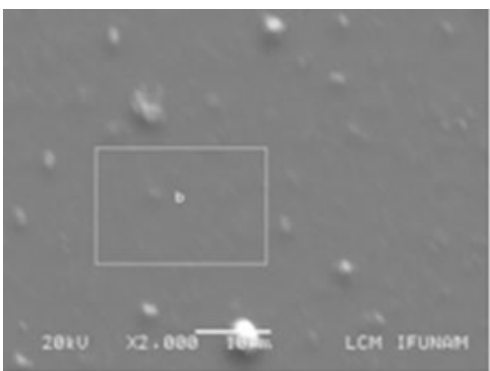

(a)

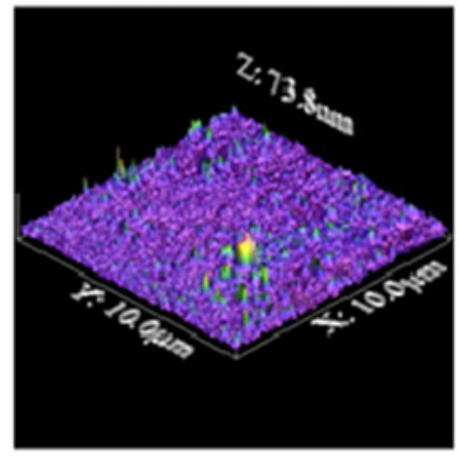

(d)

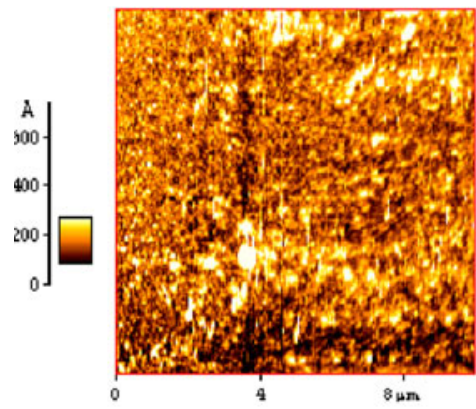

(g)

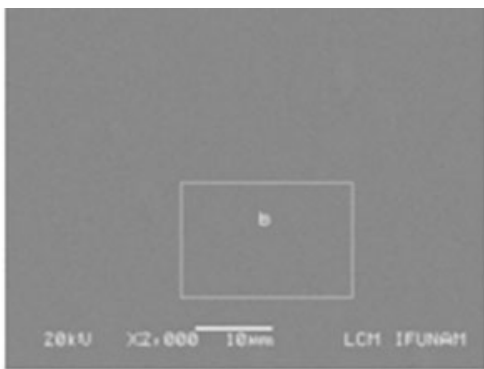

(b)

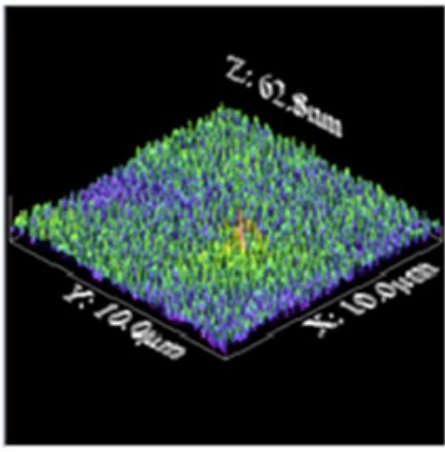

(e)

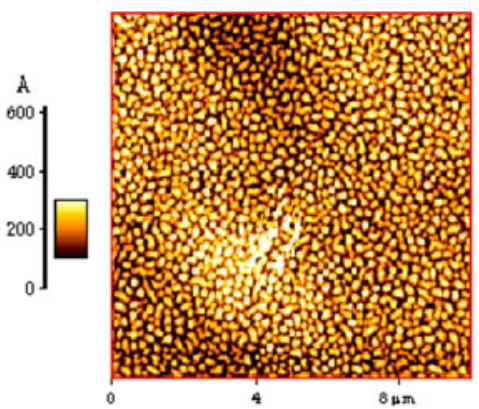

(h)

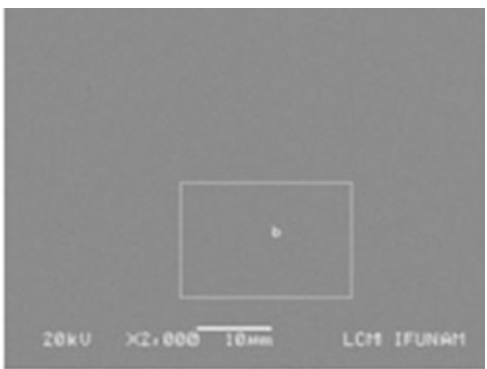

(c)

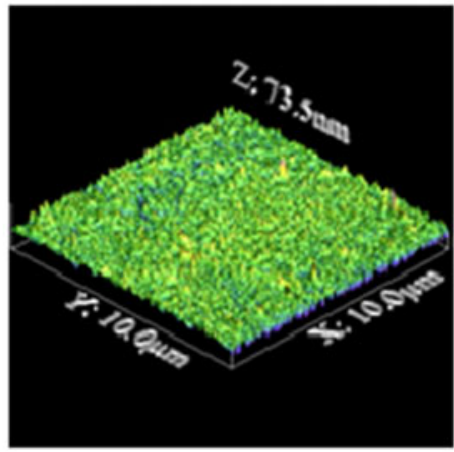

(f)

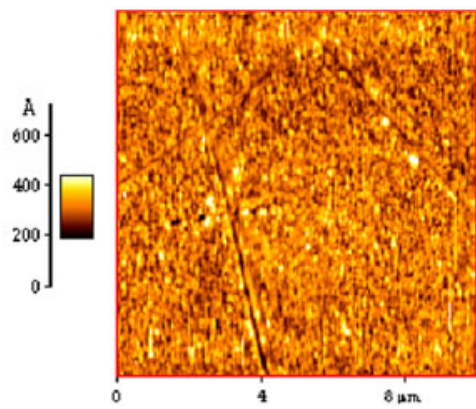

(i)

Figure 1. Morphology of NiPcTSTNa(EDA), NiPcTSTNa(BDA) and NiPcTSTNa(AqDA) films: (a-c) top view of SEM images; AFM (d-f) tapping-mode topographical and (g-i) phase images. 
films, in the 4000 to $300 \mathrm{~cm}^{-1}$ range. The UV-visible studies were performed in a Unicam spectrophotometer, model UV300, on glass substrate deposited films. Ellipsometry was carried out in a Gaertner Scientific Corporation ellipsometer (model L117), with a He-Ne laser $(630 \mathrm{~nm})$, using films deposited on silicon wafers. A four-probe press contact method was used to measure electrical conductivity of the sample within a working-temperature range between 360 and $473 \mathrm{~K}$. The temperature dependence of the electrical conductivity of the sample in this range was measured with a Keithley 230 programmable voltage source and a Keithley 485 auto-ranging pico-ammeter, both PC-controlled.

General procedure: A solution of the NiPcTSTNa salt $\left(\mathrm{C}_{32} \mathrm{H}_{12} \mathrm{~N}_{8} \mathrm{Na}_{4} \mathrm{NiO}_{12} \mathrm{~S}_{4}\right)$ in absolute ethanol was added to a solution of the appropriate diamine ligand [ethylenediamine (EDA), 1,4-diaminebutane (BDA) or 2,6-diamineanthraquinone (AqDA)] in the same solvent. The resultant mixture was maintained under reflux for about 3 days until a precipitate was obtained. The product was then filtered, washed with distilled water and absolute ethanol to eliminate unreacted diamine and phthalocyanine, respectively and then dried in vacuum.

NiPcTSTNa(EDA): $\quad 0.40 \mathrm{~g}(0.41 \mathrm{mmol})$ of $\mathrm{C}_{32} \mathrm{H}_{12} \mathrm{~N}_{8} \mathrm{Na}_{4}$ $\mathrm{NiO}_{12} \mathrm{~S}_{4}$ in $20 \mathrm{~mL}$ ethanol, $10 \mathrm{~mL}$ of ethylenedyamine (excess) in $10 \mathrm{~mL}$ ethanol. Violet powder, yield $82 \%$, m.p. $335^{\circ} \mathrm{C}(\mathrm{dec})$.

NiPcTSTNa(BDA): $\quad 0.21 \mathrm{~g}(0.21 \mathrm{mmol})$ of $\mathrm{C}_{32} \mathrm{H}_{12} \mathrm{~N}_{8} \mathrm{Na}_{4}$ $\mathrm{NiO}_{12} \mathrm{~S}_{4}$ in $15 \mathrm{~mL}$ ethanol, $0.42 \mathrm{~g}(4.8 \mathrm{mmol})$ of $1,4-$ diaminebutane in $15 \mathrm{~mL}$ ethanol. Magenta powder, yield $86 \%$, m.p. $340^{\circ} \mathrm{C}(\mathrm{dec})$.
NiPcTSTNa(AqDA): $0.20 \mathrm{~g}(0.20 \mathrm{mmol})$ of $\mathrm{C}_{32} \mathrm{H}_{12} \mathrm{~N}_{8}$ $\mathrm{Na}_{4} \mathrm{NiO}_{12} \mathrm{~S}_{4}$ in $15 \mathrm{ml}$ of ethanol, $0.40 \mathrm{~g}(1.6 \mathrm{mmol})$ of 2,6-diamineanthraquinone in $15 \mathrm{~mL}$ ethanol. Violet powder, yield $72 \%$, m.p. $340{ }^{\circ} \mathrm{C}$ (dec).

\section{Results and discussion}

Surfaces of NiPcTSTNa(EDA), NiPcTSTNa(BDA) and NiPcTSTNa(AqDA) samples were scanned by AFM and SEM. Topography obtained by AFM showed roughness, RMS, with a wrinkle height of $66.4 \mathrm{~nm}$ for NiPcTSTNa(EDA); $39 \mathrm{~nm}$ for NiPcTSTNa(BDA) and $49.9 \mathrm{~nm}$ for NiPcTSTNa(AqDA). The difference in roughness between these three samples could be correlated with type of amine in each molecular material (Ottaviano et al 1997). These non-crystalline thin films showed homogeneity in a continuum media, which were steady along surface for each sample. These features were substantiated by typical top-view SEM images (figures 1a-c) and compound analysis, EDS. $3 \mathrm{D}$ topography and phase images at $10 \times 10 \mu \mathrm{m}$ (figures $1 \mathrm{~d}-\mathrm{f}$ and $1 \mathrm{~g}-\mathrm{i}$ ) reveal thin films spreading where the presence of granular aggregates is observed.

An EDS analysis was performed to determine the chemical composition of the novel molecular materials. Figure 2 shows EDS spectrum for NiPcTSTNa(AqDA), where presence of the reference elements for both donor and acceptor species is observed. Analogous results were obtained for other compounds.

A comparison of IR absorption spectra of powder and thin film forms deposited on fresh cleavage $\mathrm{KBr}$ single crystal indicated that the thermal evaporation technique is a good technique to obtain NiPc stoichiometric thin films (El-Nahass et al 2005b). IR spectra of the powder samples show characteristic absorption bands at 1560, 1140, 1068,

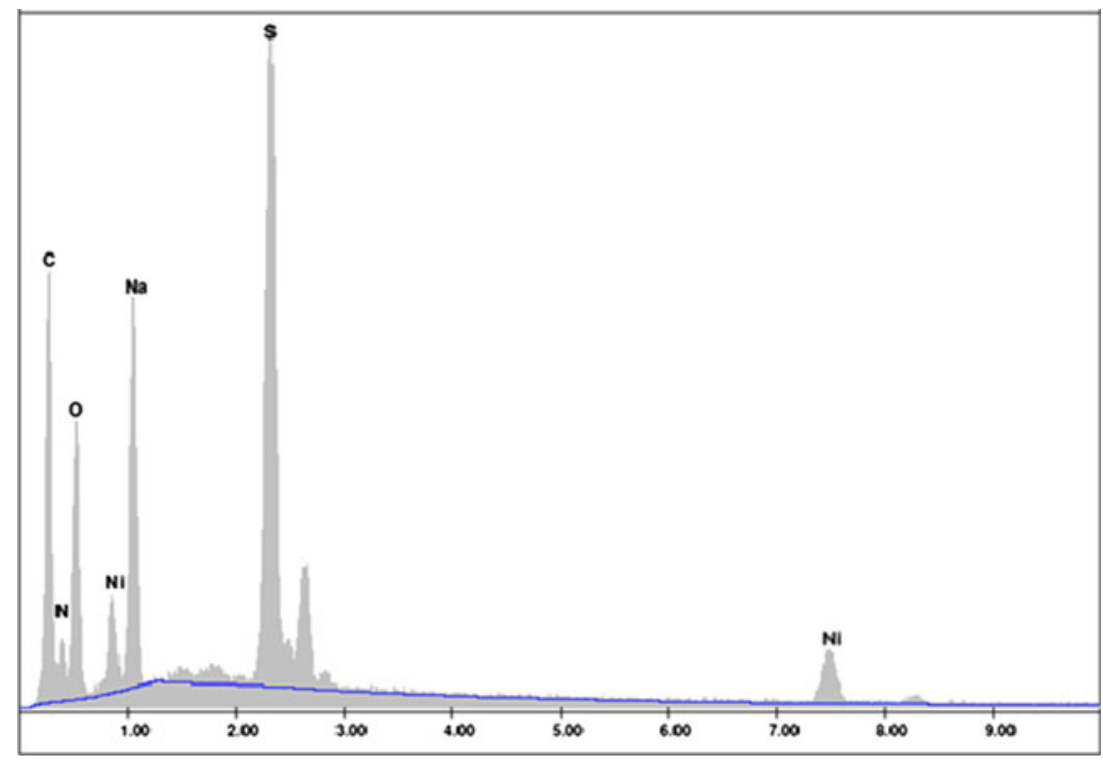

Figure 2. EDS spectrum for NiPcTSTNa(AqDA). 
970, 885 and $740 \mathrm{~cm}^{-1}$, and around 1330 and $1060 \mathrm{~cm}^{-1}$ of the original metal phthalocyanine-tetrasulfonate. Also, $\mathrm{N}-\mathrm{H}$ stretching bands around 3400 and $3335 \mathrm{~cm}^{-1}$ indicate the presence of $\mathrm{NH}_{2}$ and $\mathrm{NH}$ groups. The band around $1728 \mathrm{~cm}^{-1}$ corresponds to $\mathrm{N}-\mathrm{H}$ bending vibrations and that at $2940 \mathrm{~cm}^{-1}$ to $\mathrm{C}-\mathrm{H}$ stretching vibrations of the aliphatic chain of the amine ligands in NiPcTSTNa(EDA) and NiPcTSTNa(BDA). Additionally, NiPcTSTNa(AqDA) exhibits a strong $\mathrm{C}=\mathrm{O}$ stretching vibration band at $1628 \mathrm{~cm}^{-1}$. The peaks in the $700-400 \mathrm{~cm}^{-1}$ interval originate most probably from vibrations in the benzene ring in interaction with the pyrrole ring. The main peak at $730 \mathrm{~cm}^{-1}$ is attributed to non-planar deformation of $\mathrm{C}-\mathrm{H}$ bonds of benzene rings (El-Nahass et al 2005b). The medium band at $755 \mathrm{~cm}^{-1}$ and a band at $779 \mathrm{~cm}^{-1}$ also correspond to non-planar vibrations of the C-H bonds (El-Nahass et al 2005b).

The UV-visible spectra in the 200-1150 nm wavelength range for the NiPcTSTNa(L) films, obtained at room temperature, are shown in figure 3 . The bands originate from molecular orbitals within the aromatic $18 \pi$ electron sys- tem and from overlapping orbitals on the metal central atom (Ottaviano et al 1997; El-Nahass et al 2005a; Seoudi et al 2006). The band of the phthalocyanine molecule, viz $Q$ band, appears in the region between 550 and $750 \mathrm{~nm}$. The distinct characteristic peaks of $\mathrm{NiPcTSTNa}(\mathrm{L})$ in the visible region have been generally interpreted in terms of $\pi-\pi^{*}$ excitations between bonding and antibonding molecular orbitals. The energy peak of the $\mathrm{Q}$ band has been explained in different ways: as a second $\pi-\pi *$ transition, as an excitation peak, as a vibrational internal interval and as a surface state (Ottaviano et al 1997; El-Nahass et al 2005a; Seoudi et al 2006). The Q-band is associated with the amine coordination with the metallic ion in phthalocyanine. The presence of this absorption band may be interpreted as an overlap of $\pi$ orbitals through the bidentate ligand. The conjugated double bonds within the structure of the films create electron orbitals overlapping between the molecules ( $\pi$ orbitals). Electrons are, therefore, able to transfer energy throughout the structure and become responsible for the absorption spectra (Sánchez Vergara et al 2008).

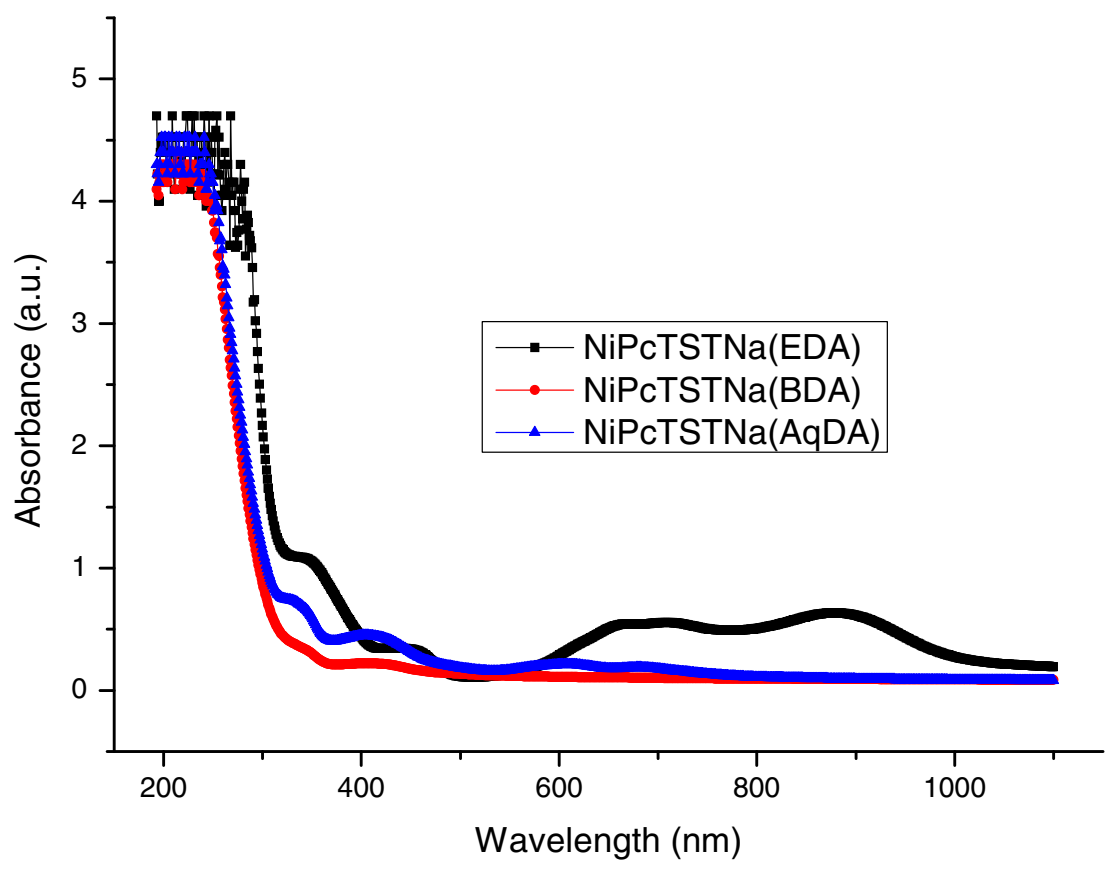

Figure 3. Electronic absorption spectra for NiPcTSTNa(EDA), NiPcTSTNa(BDA) and NiPcTSTNa(AqDA) thin films.

Table 1. Thickness, refractive index, optical activation energy and electrical parameters for NiPcTSTNa(EDA), NiPcTSTNa(BDA) and NiPcTSTNa(AqDA) thin films.

\begin{tabular}{lccc}
\hline Thin film parameter & NiPcTSTNa(EDA) & NiPcTSTNa(BDA) & NiPcTSTNa(AqDA) \\
\hline Film thickness $(\AA)$ & 320 & 1684 & 1355 \\
Refraction index & $1 \cdot 75$ & $1 \cdot 63$ & 1.585 \\
Optical activation energy $(\mathrm{eV})$ & $3 \cdot 78$ & $3 \cdot 82$ & $3 \cdot 88$ \\
Electrical conductivity at $25^{\circ} \mathrm{C}(\mathrm{S} / \mathrm{cm})$ & $2 \cdot 2 \times 10^{-3}$ & $1 \cdot 5 \times 10^{-3}$ & $4.5 \times 10^{-2}$ \\
Electrical activation energy $(\mathrm{eV})$ & $3 \cdot 7$ & $3 \cdot 3$ & $3 \cdot 7$ \\
\hline
\end{tabular}


The refractive index, $n$ (table 1 ), which was used to calculate the reflectance percentage from (1), was obtained from ellipsometry measurements.

$$
R=\frac{100(n-1)^{2}}{(n+1)^{2}} .
$$

The reflectance percentages of NiPcTSTNa(EDA), NiPcTSTNa(BDA) and NiPcTSTNa(AqDA) were 7\%, 5.9\% and $5 \%$, respectively. On the basis of these results, use of Tauc's (1972) model to interpret the energy dependence of the absorption spectra of the deposited thin films seems valid. The two main conditions to apply this model are: (i) the semiconductor material must be amorphous and (ii) the values for the estimated reflectance must be below $15 \%$.

The spectral distribution of the absorption coefficient, $\alpha$, for the deposited $\mathrm{NiPcTSTNa}(\mathrm{L})$ thin films is shown in figure 4. As observed, the behaviour is similar to that of the absorption spectra. The optical absorption coefficient describes the depth of penetration of radiation into a bulk solid. The absorption coefficient is defined by the Beer-Lambert Law and can be calculated from the optical transmittance.

$$
\alpha=-\ln T / t,
$$

where $T$ is the transmittance, related to the absorbance $A$ by $A=-\log (T), t$ the film thickness. The thickness of the films ranged between 320 and $1684 \AA$ (table 1).

The optical bandgap was determined from the analysis of the spectral dependence of the absorption near the fundamental absorption edges. In the above two regions the absorption coefficient, $\alpha$, is well described by the Urbach's relation:

$$
\alpha h v=\beta\left(h v-E_{\mathrm{g}}\right)^{n},
$$

where $h v$ is the energy of incident photons and $E_{\mathrm{g}}$ the value of the optical bandgap corresponding to transitions indicated by a value of $n$. The factor, $\beta$, depends on the transition probability and can be assumed to be constant within the optical frequency range. Plots of $\alpha^{1 / 2}$ vs $h v$ near the absorption edge of the $Q$ band for the deposited films produce a linear fit over a wider range in $h v$ as shown in figure 5 . This is the characteristic behaviour of indirect transitions in amorphous semiconductors. In this kind of materials, the optical transitions are dominated, to a first approximation, by the so-called indirect transitions. In these electronic transitions from states in the valence band to states in the conduction band, there is no conservation of the electronic momentum (Cody 1984). This type of transitions is in agreement with Kumar et al (2000) for free and rare-earth complexed pthalocyanine and in disagreement with Collins et al (1993), Ambily and Menon (1999), and El-Nahass et al (2001) for $\mathrm{PbPc}, \mathrm{CuPc}$, and FePc thin films, respectively. The electron transport in the films reported in this work is strongly influenced by their molecular structures and the generation of Frenkel-type, tightly-bound excitons (Burns 1990). It has been noticed (Hill et al 2000) that significant charge localization in organic molecular materials leads to a significant difference between size of the optical gap and size of the transport gap, which corresponds to the energy of formation of a separated free electron and a hole. Whereas the optical

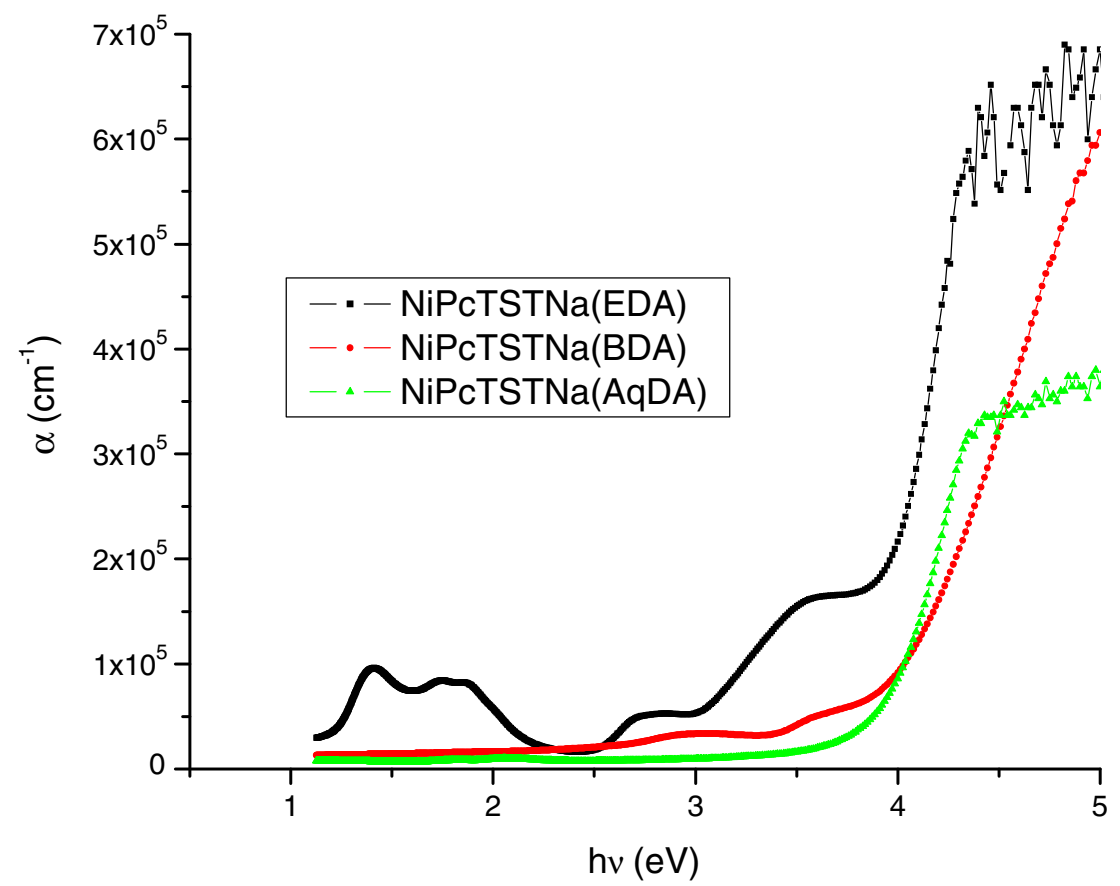

Figure 4. Plot of $\alpha$ vs $h v$ of NiPcTSTNa(EDA), NiPcTSTNa(BDA) and NiPcT$\mathrm{STNa}(\mathrm{AqDA})$ thin films. 


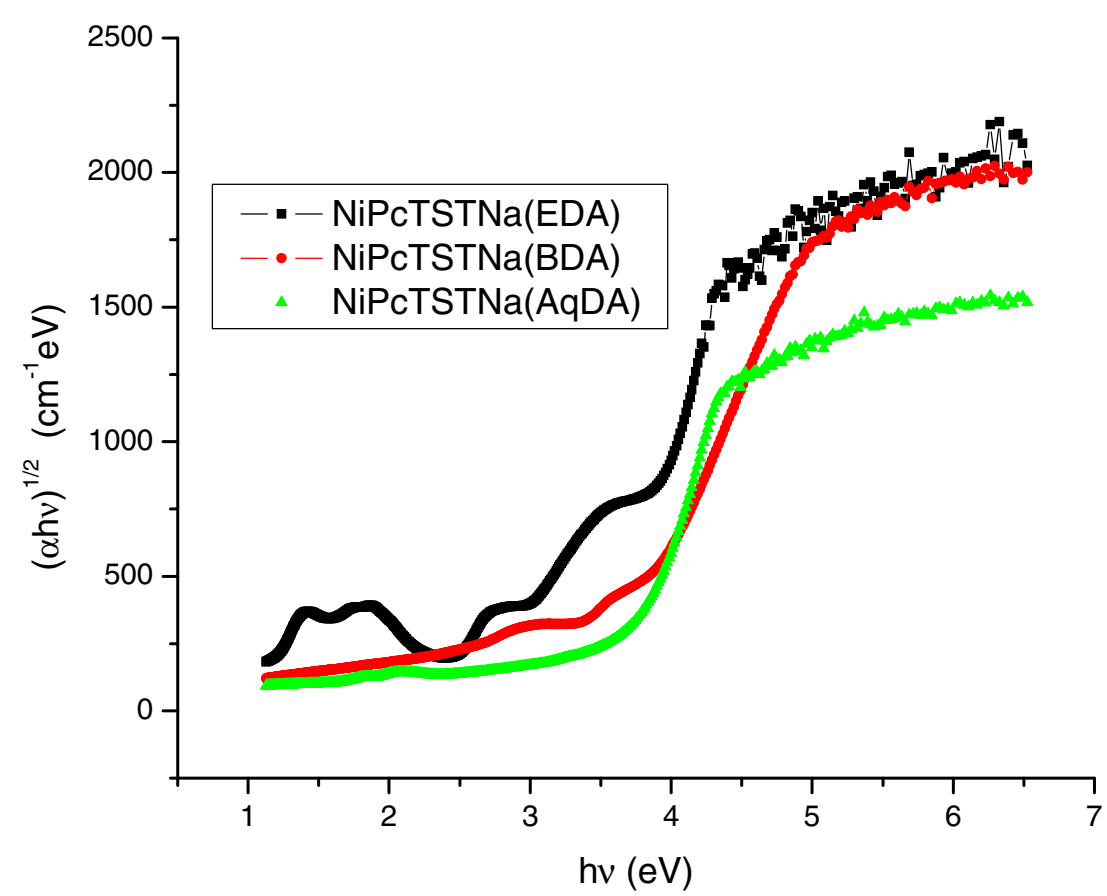

Figure 5. Plot of $(\alpha h v)^{1 / 2}$ vs $h v$ of NiPcTSTNa(EDA), NiPcTSTNa(BDA) and NiPcTSTNa(AqDA) thin films.

gap can be measured by optical absorption spectroscopy, the transport gap can be measured by ultraviolet or inverse photoemission spectroscopy and it is larger than the optical gap by a quantity equal to the binding energy of the Frenkel excitons. The energy gaps obtained are listed in table 1 and do not show important differences. This may be attributed to the fact that the charge transport in the materials is mainly related to the metal phthalocyanine, independently from the amine ligand. If it is considered that the optical activation energy values for semiconductors are located in the range between 1 and $3 \mathrm{eV}$, it can be inferred that the obtained thin films show a quite semiconductor-like behaviour, as their optical activation energies are around $3.8 \mathrm{eV}$.

The variation of electrical current with temperature was evaluated by using the four-probe method. This is one of the most used techniques for resistivity measurements in the semiconductor industry. Such tests are made on a line along the film, with equal spaces between the selected points; also, the current flow must be low enough to prevent sample heating, the voltmeter must have a high input impedance and measurements must be performed for several contact points, so that any injected minority charge carrier recombines. Temperature was measured by means of a chromel/alumel thermocouple mounted in close proximity to the specimen of interest. Electrical characterization was performed over a voltage range appropriate to the thickness of the sample (Abdel-Malik et al 1995; Shafai and Anthopoulos 2001). Figure 6 shows typical temperature dependence of the electrical conductivity of the films during heat treatment. By analysing the shape of the $\ln \sigma=f\left(10^{3} / T\right)$ plots, useful information regarding the processes occurring in the samples during treatment can be obtained; additionally, it provides support to the consideration that the model based on the bandgap representation could explain the electronic transfer mechanism in the films. Therefore, the estimate of some characteristic parameters of these materials has been made by using the expressions deduced for the intrinsic conduction domain of semiconductors. From figure 6, electrical conductivity of each material was evaluated at $25^{\circ} \mathrm{C}$. The results are shown in table 1 . The NiPcTSTNa(AqDA) material shows highest conductivity at ambient temperature. It can also be observed that the electrical conductivity values for all the films are within the range for semiconductor materials $\left(10^{-6}\right.$ to $\left.10^{1} \Omega^{-1} \mathrm{~cm}^{-1}\right)$ (Sánchez Vergara et al 2008). This is an important fact, since a molecular semiconductor is generally defined in terms of its room temperature conductivity and its behaviour with temperature.

It is known that the temperature dependence of electrical conductivity, $\sigma$, for a semiconductor material in its intrinsic conduction domain, is described by the following law (Sánchez Vergara et al 2008):

$$
\sigma=\sigma_{m} \exp \left(-\frac{\Delta E_{m}}{K T}\right)
$$

where $\sigma_{m}$ is the pre-exponential factor and $\Delta E_{m}$ the activation energy for electrical conductivity. Calculated values of $\Delta E_{m}$ are presented in table 1 . Such values are similar to those obtained for the optical bandgap. This fact suggests that $\Delta E_{m}$ is an activation energy involving both the energy necessary to excite electrons from the localized states toward extended states through the mobility edge and the electrical conduction by means of hopping mechanism between localized states. 


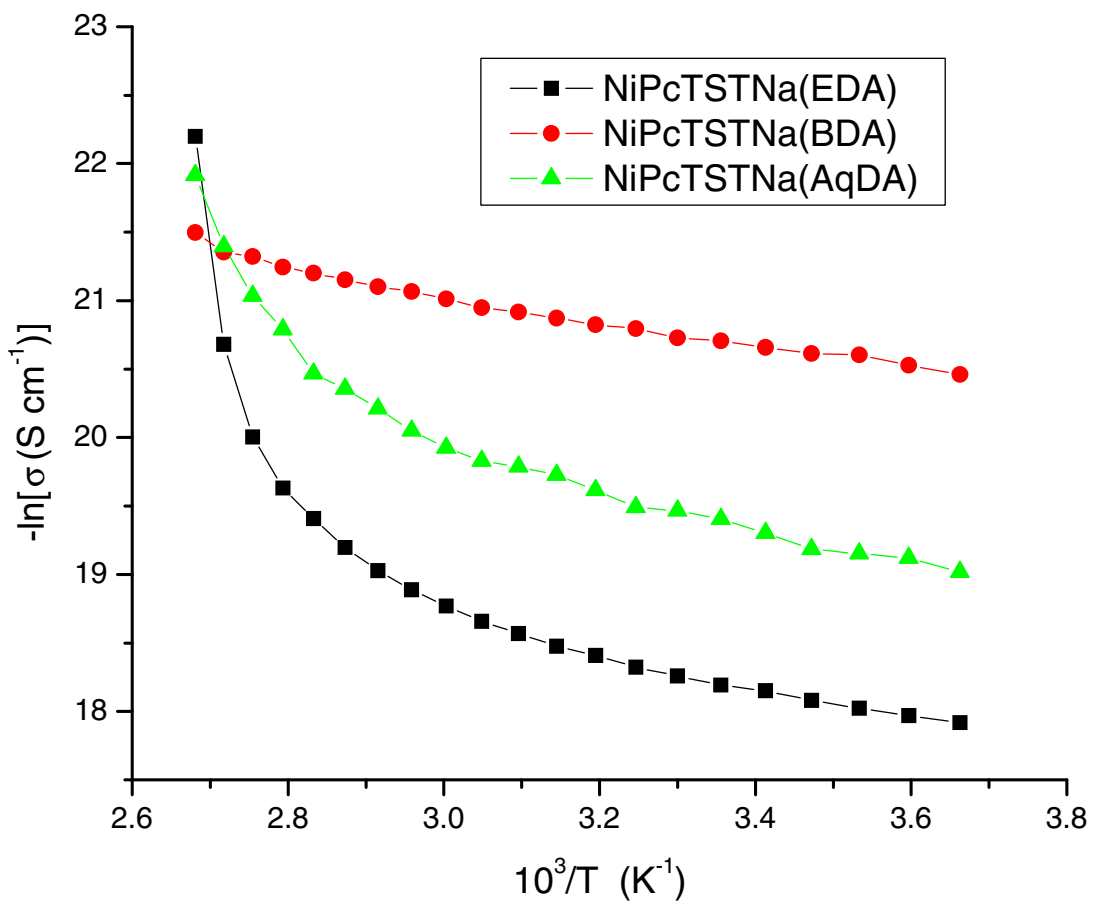

Figure 6. Temperature dependence of electrical conductivity of NiPcTSTNa(EDA), NiPcTSTNa(BDA) and NiPcTSTNa(AqDA) thin films.

Therefore, considering the optical and electrical properties of the deposited molecular films, their application in electronic and opto-electronic devices seems quite feasible.

\section{Conclusions}

Thin films of molecular materials of the NiPcTSTNa $(\mathrm{L})$ type were deposited by vacuum thermal evaporation. According to their FT-IR spectra, they are formed by the same chemical units as those of the corresponding synthesized powders. Thus, the thermal evaporation process can be, in general, considered as a molecular process. The optical characterization of the deposited thin films was performed in the 200-1150 nm spectral range. The spectral distribution of the absorbance and the absorption coefficient of the NiPcTSTNa(L) films characterized by distinct peaks in the visible region has generally been interpreted in terms of $\pi-\pi^{*}$ excitations. It is considered that $\pi-d$ transitions are involved since $\operatorname{NiPcTSTNa}(\mathrm{L})$ has a partially occupied $d$-band. NiPcTSTNa(L) films show typical semiconducting characteristics. The electron transport in these materials is strongly influenced by the phthalocyanine. The model based on the bandgap representation was successfully used to explain the electron transfer mechanism in the compounds studied. The material, NiPcTSTNa(AqDA), has the highest conductivity, and in general, the electrical conductivity values at room temperature for all the compounds are within the range for semiconductor materials $\left(10^{-6}\right.$ to $\left.10^{1} \Omega^{-1} \mathrm{~cm}^{-1}\right)$. On the basis of the optical bandgap values, magnitude of the electrical conductivities and feasibility of preparing these compounds as thin films, it can be concluded that these materials may have a potential use in electronic device fabrication.

\section{References}

Abdel-Malik T G, Abdel-Latif R M, El-Samahy A E and Khalil S M 1995 Thin Solid Films 256139

Ambily S and Menon C S 1999 Thin Solid Films 347284

Bialek B, Kim I G and Lee J I 2002 Synth. Met. 129151

Burns G 1990 Solid state physics (San Diego: Academic Press, Inc.) Campidelli S et al 2008 J. Am. Chem. Soc. 13011503

Cody G D 1984 Hydrogenated amorphous silicon, Part B, Optical properties, semiconductors and semimetals, J I Pankove (ed.), (Orlando: Academic Press) Vol. 21

Collins R A, Krie A and Abass A K 1993 Thin Solid Films 229113 Davidson A T 1992 J. Chem. Phys. 77168

de la Torre G, Vazquez P and Torres T 2004 Chem. Rev. 1043723

Del Caño T, Parra V, Rodríguez M L, Méndez R F, Aroca R F and De Saja J A 2005 Appl. Surf. Sci. 246327

El-Nahass M M, Soliman H S, Farid H S, Farag A A M and El-Shazly A A 2001 J. Opt. 30121

El-Nahass M M, Farag A M, Abd-El-Rahman K F and Darwish A A A 2005a Opt. Laser Technol. 37513

El-Nahass M M, Abd-El-Rahman K F and Darwish A A A 2005b Mater. Chem. Phys. 92185

Hill I G, Kahn A, Soos Z G and Pascal R A Jr 2000 Chem. Phys. Lett. 327181

Kumar G A, Thomas J, George N, Kumar B A, Shnan P R, Poori V P N, Vallabhan C P G and Unnikrishanan N V 2000 Phys. Chem. Glasses $\mathbf{4 1} 89$ 
Ottaviano L, Di Nardo S, Lozzi L, Passacantando M, Picozzi P and Santucci S 1997 Surf. Sci. 373318

Sánchez Vergara M E, Ortiz Rebollo A, Alvarez J R and Rivera M 2008 J. Phys. Chem. Solids 691

Schmeisser D, Rager A, Thonke K, Pilkuhn M, Frohlich D, Gaauglits G, Schafer M and Oelkrug D 1991 Synth. Met. 41 1457
Seoudi R, El-Bahy G S and El Sayed Z A 2006 Opt. Mater. 29304 Shafai T S and Anthopoulos T D 2001 Thin Solid Films 398361

Tauc J 1972 'Optical properties of non-crystalline solids', in Optical properties of solids, F Abele's (ed.), (Amsterdam: NorthHolland Publishing Co.) p. 277

Wojdyla M, Bala W, Derkowska B, Rebarz M and Korcala A 2008 Opt. Mater. 30734 